\title{
An approach to categorize chest $X$-ray images using sparse categorical cross entropy
}

\author{
Chaithanya B. N., Swasthika Jain T. J., A. Usha Ruby, Ayesha Parveen \\ Department of CSE, Gitam School of Technology, Nagandenahalli, India
}

\begin{tabular}{l}
\hline \hline Article Info \\
\hline Article history: \\
Received Jul 8, 2021 \\
Revised Oct 19, 2021 \\
Accepted Oct 26, 2021 \\
\hline
\end{tabular}

\section{Keywords:}

COVID-19

Pneumonia viral

Prediction

Sparse categorical crossentropy

Stochastic gradient descent

\begin{abstract}
The Coronavirus disease (COVID-19) pandemic is the most recent threat to global health. Reverse transcription-polymerase chain reaction (RT-PCR) testing, computed tomography (CT) scans, and chest X-ray (CXR) images are being used to identify Coronavirus, one of the most serious community viruses of the twenty-first century. Because CT scans and RT-PCR analyses are not available in most health divisions, CXR images are typically the most time-saving and cost-effective tool for physicians in making decisions. Artificial intelligence and machine learning have become increasingly popular because of recent technical advancements. The goal of this project is to combine machine learning, deep learning, and the health-care sector to create a categorization technique for detecting the Coronavirus and other respiratory disorders. The three conditions evaluated in this study were COVID-19, viral Pneumonia, and normal lungs. Using X-ray pictures, this research developed a sparse categorical cross-entropy technique for recognizing all three categories. The proposed model had a training accuracy of $91 \%$ and a training loss of 0.63 , as well as a validation accuracy of $81 \%$ and a validation loss of 0.7108 .
\end{abstract}

This is an open access article under the CC BY-SA license.

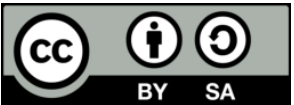

\section{Corresponding Author:}

Chaithanya B. N.

Department of CSE, Gitam School of Technology

NH 207, Nagadenehalli, Doddaballapura, Karnataka 561203, India

Email: cnagaraj@gitam.edu

\section{INTRODUCTION}

The world has recently witnessed how the coronavirus infection epidemic, which has resulted in the severe acute respiratory syndrome Coronavirus 2 (SARS-CoV-2) infecting individuals across the world, is spreading [1]. The Coronavirus disease, which is highly contagious, was first brought to the notice of the Chinese authorities in Wuhan in December 2019, and it has affected over 25 million people worldwide. Coronavirus is a global hazard to both people and technologies [2]. COVID-19 illness is more likely in individuals with poor immunity, old age, and medical problems, especially those involving the lungs. Cough, cold, high fever, and breathing problems are all signs of COVID-19. COVID-19 prevention methods include frequent hand washing, avoiding touching the mouth, eyes, nose, and face, and maintaining a social distance from others. Pneumonia has been the most common and severe event, and serious problems, such as acute respiratory distress syndrome (ARDS), can develop quickly, leading to COVID-19's high mortality rate [3]-[5]. Because there are a limited number of specialists qualified to acquire Coronavirus diagnosis expertise, there has been a coordinated push to use artificial intelligence (AI), particularly deep-learningbased techniques, in reasonably accurate Coronavirus epidemics prediction and diagnosis, where wellannotated data are frequently required [6]. 
The diagnosis and observation of these respiratory events are guided mainly by chest X-rays and chest computed tomography scans. In some instances, CT scans revealed inconsistent results in patients before symptoms develop and before the viral ribonucleic acid (RNA) was detected [7]-[9]. X-rays have proven helpful in the initial stages of diagnosing then screening affected for COVID-19 [10].

COVID-19 has reached epidemic level, and the number of patients is rapidly growing, thus extensive diagnostic surveillance is critical for recognizing and controlling the spread of this rapidly spreading illness. According to studies, COVID-19 is a potentially fatal virus, and viral testing is critical for early diagnosis and management of the disease to establish how so many people were affected and how many others are at risk. Chest tomography scanning, comprising a CT scan and X-rays, are the most prevalent diagnostics for COVID-19 diagnosis [11]. These may help in the early identification and diagnosis of this illness, in addition to reverse transcription polymerase chain reaction (RT-PCR), isothermal nucleic acid amplification, and antigen tests. The most major respiratory organ is damaged and established 10 to 12 days after the virus assaults, according to a comprehensive analysis of CT scans of respiratory system and pulmonary of patients with COVID-19 respiratory disease. Because RT-PCR testing takes longer to determine, clinicians believe that fast and early diagnosis of clinical X-ray testing will assist establish whether a patient must be kept segregated under observation until the results of the lab tests are obtained. Detection of the disease sooner via X-rays prevents it from rapidly spreading to others during the timeframe. The examination of chest X-rays is a distinguishing feature. Patients can go back to the house if their chest $\mathrm{X}$-ray picture is normal, and they are awaiting the results of their laboratory testing. That is where our contribution to this study is significant. COVID-19 is usually diagnosed using at least one of the three measures. At computed tomography, scan-based measurements are made by analyzing 3D X-rays from multiple angles.

Most hospitals do not have the necessary equipment for this assessment. It takes around fifteen minutes each patient, in addition to the time it takes for CT disinfection. In phlegm or a nasopharyngeal swab, the RT-PCR test detects viral RNA [12]. It necessitates specialized materials and supplies that aren't readily available. It takes at least 12 hours, which isn't ideal as a good outcome. COVID-19 patients should be identified and followed up on as soon as feasible. According to several studies, the results of numerous RT-PCR tests done on the same patient at different periods of the illness are inconsistent, resulting in a significant false-negative rate [13]. Chowdhury et al. employed image augmentation and a transfer learning method to train and validate numerous pre-trained deep convolutional neural networks (CNNs). The networks were taught to distinguish between two types of pneumonia: i) normal and COVID-19 pneumonia; and ii) normal, viral, and COVID-19 pneumonia with or without image augmentation. Both methods' classification accuracy, precision, sensitivity, and specificity were calculated in accordance [14].

Our objective is to develop a categorization system that will allow us to categorize patient chest X-ray images into positive and negative COVID-19 categories. Deep learning (DL) models for automated image processing have a lot of potential for enhancing the utilization of chest X-ray (CXR) images in COVID-19 detection. A DL model that is both dependable and exact can be utilized as a diagnostic tool and to help in medical decision-making. A chest CT scan or X-ray can reveal ground-glass opacity, bilateral flaws, and interstitial irregularities in CXR and CT images. Detecting abnormalities and indications in X-ray images, though, could be challenging without professional medical analysis [15], [16]. Computer-aided diagnosis (CAD) has swiftly evolved to assist clinicians discover and quantify the essential organs of diagnosed illnesses using X-ray images, allowing them to overcome these restrictions. Furthermore, recent research utilizing artificial vision and deep learning techniques to analyze Chest X-ray images have yielded promising results. The contributions of this work are summarized in the following sections.

- We create a COVID-19 CXR database by altering and merging 3 free access Chest X-ray data and use deep learning methods.

- Developing a deep learning network to aid in the identification and categorization of COVID-19 positive individuals.

- Tests have shown that the suggested model performs better in categorization.

\section{RESEARCH METHOD}

Peeking at some of the related research for detecting pneumonia and COVID-19 will be covered in this section. To identify COVID-19 spreading between people, Narin et al. analyzed three ResNet50, Inception-ResNetV2, and InceptionV3 versions of COVID-19 using CNN-based system and performed an alternative diagnosis using their automated detection method [17]. To detect Coronavirus respiratory illnesses, they utilized X-rays on infected patients. InceptionV3 and Inception-ResNetV2 have the lowest classification accuracy when compared to ResNet50. 
Researchers collected 453 CT images of pathogen-confirmed COVID-19 for publication in [18]. 217 of the collected images served as training data, and the system was constructed using the inception migration modeling approach. Based on the internal validation, the precision and sensitivity of the external dataset was 73.1 percent, the accuracy of 80.5 percent, and the dependability of 80.5 percent.

During the illness, Wang et al. [19] observed abnormalities in 51 of 64 patients' chest X-rays (CXRs). RT-PCR was used to confirm the COVID-19 infection. The most common outcome on baseline CXR was consolidation (47\%), followed by ground-glass opacity (GGO) (33\%). Periphery involvement $(41 \%)$ and lower spread zone involvement $(50 \%)$ were most common, and most individuals had bilateral involvement $(50 \%)$ as well. Among the $58(91 \%)$ individuals with a positive initial RT-PCR, $38(59 \%)$ had abnormal CXR results. CXRs revealed abnormalities in six $(9 \%)$ of the individuals whose RT-PCRs were negative. A 48-hour test results in five positive results from six patients, and one positive result from another patient. The first RT-PCR detection rate was 58/64, with a sensitivity of 91 percent (95 percent CI [20]), which was better than the first CXR detection rate (44/64, with a sensitivity of 69 percent, 95 percent CI [21] $(\mathrm{p}=0.009)$.

Wong et al. [22], who studied a bigger organization of 1014 sufferers and discovered that CT had a ninety-seven-percentage sensitivity in detecting COVID-19, exhibited flaws like the ones discovered withinside the Raptis et al. look at [23]. The affected person populace withinside the look at with the aid of using $\mathrm{Ai}$ and co-workers become now no longer specified. Nonetheless, we decided that the pattern protected hospitalized sufferers, who can be much more likely than outpatients to have strange CT results [24]. As in Fang and colleagues' study, CT turned into hired as a binary measure, and there has been no set up criterion for comparing nice findings. The imaging examples used by the authors found out focal floor opacities, which can be visible in quite a few illnesses. Because those sufferers are believed to provide promising CT outcomes, sensitivity is exaggerated, and accuracy is compromised. The handiest good-sized distinction among the 2 experiments changed into that AI and colleagues' evaluation used a higher affected person group, main the reader to consider that the cohort and look at layout have been pleasant appropriate to check sensitivity when, in fact, the cohort and method used for this motive have been simply as limited as Fang and colleagues. Both scientists made the identical mistakes and got here to the identical vast conclusions.

The training purpose is to decrease the common possibility error among the target and predicted label for every pixel, as defined through A. Ruby et al. [25] to enhance ImageNet class accuracy even more, the Inception V3 structure is utilized. The cautioned technique gives the exceptional outcomes, in keeping with the results. Karhan et al. proposed the RESNet50 model for the radiological images of the chest and Reverse Transcription-Polymerase Chain Reaction assessment for diagnosing COVID-19 patients. It was categorized with the ResNet50 model, a kind of CNN architecture is used for detecting COVID-19 patients using chest X-ray images [26]. Ilshat Garafiev et al. the research looked at how first-year master's students assessed the challenges with online education during the COVID-19 pandemic. It was proposed that difficulties can be divided into two categories. The first category includes issues that arise because of participants in the educational process being unable to communicate effectively online. There are also issues with technical challenges that arise when taking part in online schooling [27].

Hayfa et al. an evaluation of AMAN (Jordan's COVID-19 tracking mobile appuser)'s experience (UX) using a questionnaire instrument was proposed in this study. To gather input and determine UX, the study will examine how users interact and use the monitoring app. The information was gathered from 1208 people who had used the app before; an online questionnaire was created and circulated through social media groups [28]. Shamrat et al. The pandemic has engulfed the entire world. Almost the entire planet fell into lockdown to protect the population from the lethal COVID-19. Several vaccinations for the virus have been developed by scientists all around the world. Pfizer, Moderna, and AstraZeneca are three of the most wellknown. However, the public has used social media such as Twitter to voice their concerns about the vaccinations' safety and efficacy. Twitter application programming interface (API) authentication tokens are being used in this investigation to extract such tweets from the service. NLP is used to store and process unprocessed tweets. Unsupervised KNN classification algorithms are used to sort the cleaned data [29].

Noori et al. occasionally, patients will require additional blood as a form of compensation, such as during severe surgery or other scenarios. Transfusions of plasma are crucial in the present Coronavirus Pandemic (COVID-19). In this study, presented a donation system that coordinates appointments between donors and patients when a fresh blood donation is needed. Using Bootstrap technology, the website was built to be accessible from a personal computer (PC) or mobile web browser. The website has a big database with information about donors and their blood groups, as well as available time and other personal details to make the donation procedure easier. There are no restrictions on who can use this system and it can be utilized by any hospital, blood bank, or individual to manage the donation procedure [30].

Haglan et al. a framework was proposed to build a comprehensive monitoring system based on internet of things (IoT) technologies with respect to patient and hospital and describes what are the expected 
advantages that we get after implementing the system [31]. Reza et al. COVID-19 is the deadliest epidemic in human history. It's a highly contagious illness that can pass from person to person without causing symptoms. Because it is highly contagious, diagnosing, and isolating patients with it has become a top priority for healthcare providers. This study demonstrated an alternate method of identifying COVID-19 individuals by doing an automatic examination of the patients' chest X-rays. Six pre-trained deep learning models were used to construct such an effective system. VGG16, InceptionV3, Xception, DenseNet201, InceptionResNetV2, and EfficientNetB4 were the models used. These models were created using two opensource datasets containing chest X-rays of COVID-19 patients. EfficientNetB4 outperformed the other models on both datasets, with 96 percent and 97 percent accuracy, respectively. The empirical findings were likewise outstanding. This type of computerized system can assist us in combating the deadly viral spread [32].

\section{METHODOLOGY}

A neural network is a recent breakthrough in deep learning that is increasingly gaining traction because of its influence on adjusting numerous elements of human existence. It also puts forth a strategy for dealing with the current crisis caused by the Coronavirus illness (COVID-19), considering all technological methods. CNNs, a kind of neural network, are frequently used in medicine and have proven to be particularly useful in the current COVID-19 epidemic. Using X-ray pictures of coronavirus patients, this study shows how CNNs may be used to detect and forecast coronavirus illness. Sequential, Convolution2D, MaxPooling2D, Flatten, and Dense with 10 layers of rectified linear unit (ReLU) and SoftMax activation were used as the basis models for coronavirus detection and forecasting. Also with smaller datasets, these models showed to be effective in detecting the presence of coronavirus. Images of $\mathrm{X}$-ray, The Sparse categorical cross-entropy architecture will be utilized to identify COVID-19 positive people. The four elements of the overall architecture shown in Figure 1 are preprocessing, rescaling, max-pooling, and classification. To further validate these findings, an iterative application of the stochastic gradient descent (SGD) approach was used in the dataset to improve an unbiased function and maintain a smoothness quality for the pictures. Using a sparse categorical cross entropy for the categorization of X-ray images in the dataset, they were able to achieve a training accuracy of 91 percent. As a result of the convenience of improved medical imaging datasets, CNNs are highly useful for COVID-19 identification and forecasting.

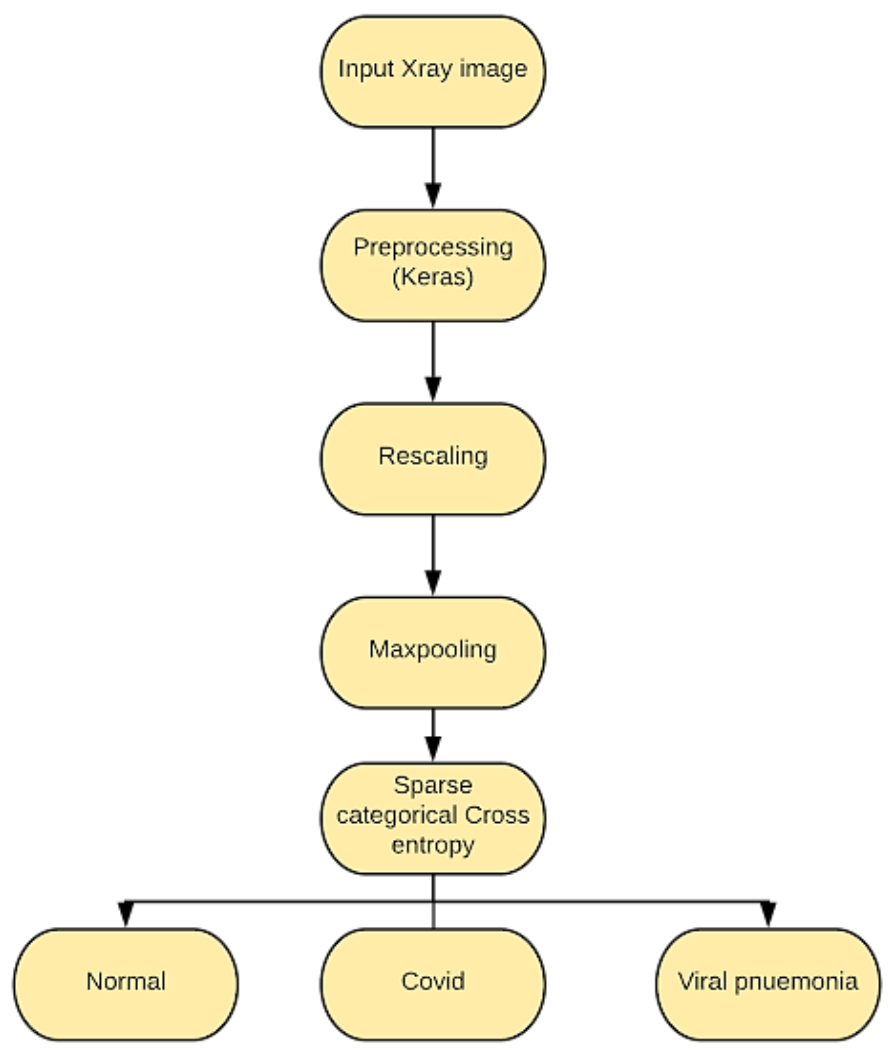

Figure 1. Flow chart of the sparse categorical cross-entropy 


\subsection{Dataset preparation}

The widely accessible COVID19-image (X-ray image) dataset was applied for experimenting our model. This dataset was taken from Kaggle. The COVID19-image (X-ray image) dataset consists of 348 COVID19-image (X-ray image) from 215 patients and 396 non-COVID19-images (X-ray image). COVID-19 impacted CT pictures from the COVID-CT dataset. The dataset was classified as COVID and non-COVID pictures, which were then transformed into groups, after analyzing the importance of images and labels for training and testing of the $\mathrm{CNN}$ classifier. These subsets are chosen randomly. To achieve satisfactory accuracy rates smaller covid19-image dataset is considered. However, as the amount of data in the dataset increases, the results are consistently enhanced.

\subsection{Preprocessing}

Pre-processing aims to improve X-ray image data, eliminate undesirable distortions, and improve specific image properties applicable to future data processing. Here the data set is preprocessed using Keras. Then Image rescaling, which is a crucial component of image development, has been done before machine learning techniques are applied. The best way to resize an image is to recreate the original continuous function, then resample it at a varying rate. In machine learning, image normalization is used to make the model training less sensitive to the characteristics' scale. This makes it possible for our model to converge to better weights, resulting in a more precise model.

The size of the feature maps is reduced by using pooling layers. The number of features which must be learnt and the amount of computations that can only be done in the neural network are both reduced as a result of this. The pooling layer brings all of the features in a region of the feature map created by the convolution layer together. As a result, instead of the precisely positioned features of the convolution layer, actions are performed on summarized features. As a result, the model is more resistant to changes in the location of features in the input picture. Applying a two-dimensional filter over each channel of the feature vector and grouping the features that lie inside the filter's covering zone is how the pooling process works. The (1) shows the percentages of output received after quite a pooling layer for a feature vector with dimensions $\mathrm{n}_{\mathrm{h}}, \mathrm{x}, \mathrm{n}_{\mathrm{w}}, \mathrm{x}, \mathrm{n}_{\mathrm{c}}$.

where,

$$
\left(\frac{\mathrm{n}_{\mathrm{h}}-\mathrm{f}+1}{\mathrm{~s}}\right) \times\left(\frac{\mathrm{n}_{\mathrm{w}}-\mathrm{f}+1}{\mathrm{~s} \times \mathrm{n}_{\mathrm{c}}}\right)
$$

nh - height of feature map

nw- width of feature map

nc- No of channels in feature map

f- Size of filter

s- Stride length

\subsection{MaxPooling2D}

The maximum pooling procedure is used for 2D spatial data. Max pooling is a type of pooling that selects the most major component from the area of the feature map defined by the filter. Therefore, the maxpooling layer will produce a feature vector that retains the key properties of the previous feature vector. Max pooling2D down samples the input image by taking the most significant value for each dimension along the features axis across the window provided by pool size. When max pooling is used in a model, it decreases the dimensionality of images by lowering the number of pixels in the preceding convolutional layer's output. After a CNN layer, a MaxPooling2D layer is frequently employed to minimize the output's intricacy and avoid overfitting the data.

An activation function is a simple function that turns inputs into outputs with a predetermined range of values. The ReLU function is a non-linear activation function shown in Figure 2. The ReLU function has the advantage over all other activation functions in that it does not excite all the neurons at the same time, and it tackles the problem of vanishing gradients, allowing models to learn faster and perform better.

$$
f(x)=\max (0, x)
$$

The outcome for negative input values is zero, indicating that the neuron is not engaged. Compared to the sigmoid and tanh functions, the ReLU function is considerably more highly scalable since only a few neurons are active. 


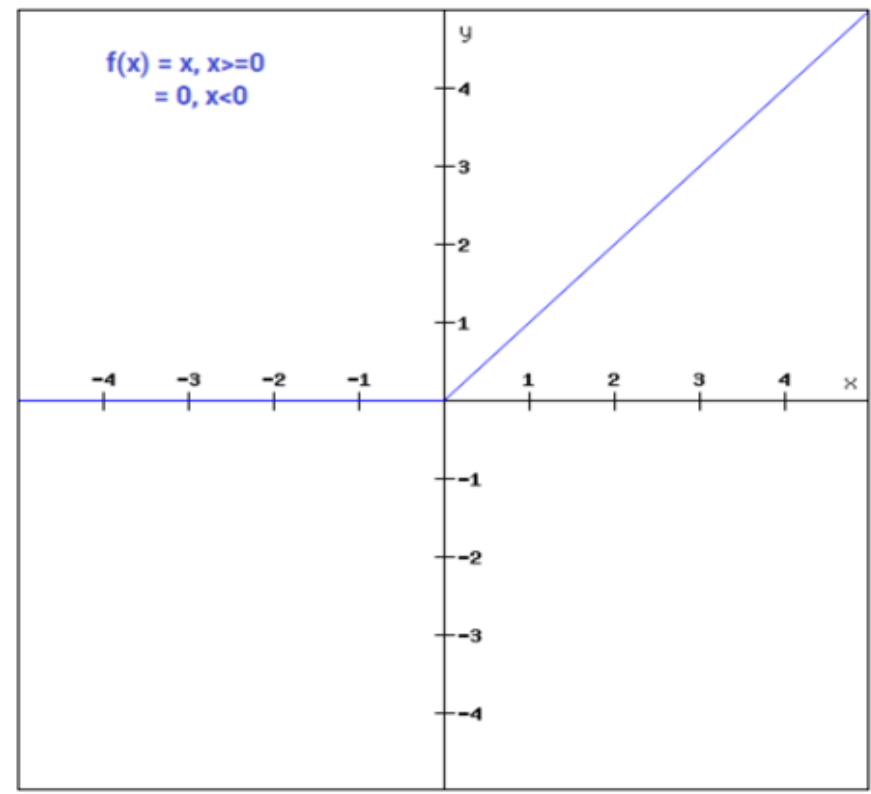

Figure 2. ReLu activation function

\subsection{SoftMax}

SoftMax transforms a real vector into a category probability vector. The output vector's members are in the range $(0,1)$ and add up to 1 . The function is helpful for classification issues, particularly multiclass classification issues, because it returns a "confidence score" for each class. Because we're working with probabilities, the SoftMax function's results will add up to one.

$$
\frac{\mathrm{e}^{\mathrm{Z}_{\mathrm{i}}}}{\sum_{\mathrm{J}=1}^{\mathrm{K}} \mathrm{e}^{\mathrm{Z}_{\mathrm{i}}}}
$$

It says to apply a typical exponential function to each element of the output layer, then divide by all the exponentials to normalize the results. This guarantees that the total of all exponentiated numbers equals one. The cross-entropy loss is used when changing model weights during training. The objective is to minimize the loss, the lower the loss, the better the model. A perfect model has zero cross-entropy loss.

The term "cross-entropy" refers to the amount of information that may be extracted from (4).

$$
\mathrm{L}_{\mathrm{CE}}=-\sum_{\mathrm{i}=1}^{\mathrm{n}} \mathrm{t}_{\mathrm{i}} \log \left(\mathrm{P}_{\mathrm{i}}\right), \text { f or } \mathrm{n} \text { classes }
$$

Where ti is the truth label and pi are the SoftMax probability for ith class.

\subsection{Sparse categorical cross entropy}

Categorical data that is sparse is referred to as Sparse categorical data. The loss of cross-entropy between labels and predictions is calculated using cross entropy. The loss of cross-entropy, also known as the loss of the $\log$, measures the performance of a model with a probability value ranging from 0 to 1 ; as the predicted probability departs from the label, the loss of cross-entropy increases. A score/loss is calibrated to punish the likelihood depending on how far it is from the expected actual value compared with the current class of intended output 0 or 1 . The penalty is inherently logarithmic, with significant differences of almost one and a tiny score of minor differences of 0 .

\subsection{Experimental setup}

The CNN methodology for the model in the covid19-image dataset is made up of convolutional, pooling, and fully connected layers. The convolutional and pooling layers extract features from the images, while the fully connected layer maps the extracted features into the output for more classification. The CT scans were reduced to a resolution of 300 x 300 pixels. The weights of the model were optimized using a four-layered CNN with an stochastic gradient descent (SGD) optimizer. The training data was set to model, the epoch value was fifteen, the ReLu function was utilized, and the soft-max activation function was employed. Because CNN can't deal with categorical data directly, the images and labels are converted to an

An approach to categorize chest $X$-ray images using sparse categorical cross entropy (Chaithanya $B . N$.) 
array with a one-hot encoder. The dataset was then divided into two sets: a training set for training the network and a validation set for model cross-validation during the training phase. The validation set kept track of the model's performance, allowing the parameters to be fine-tuned and a final model to be chosen. Python, Keras, and Tensorflow were used to create the CNN framework

\section{RESULTS AND DISCUSSION}

The understanding of medical images is examined by specialists in the medical field, such as radiotherapists and doctors. As medical records vary considerably from one patient to another differing on the infection, the objective of diagnosing the disease becomes tedious. Further, the understanding of medical images is exceptionally reliant on medical experts who have constraints in terms of skills, quickness, and exhaustion. With the present scenario, the capability of the medical care structure to deal with the pandemic has been examined. One way to attain this is to perform medical image evaluation via a computerized, accurate, and inexpensive automated framework such as a neural network model, which can produce the precisions of a skilled human intellect. The significant of medical image evaluation that an effective neural network can operate at any provided time is considerably larger than that of a human intellect.

The Sequential model is basic (just a list of layers), but it is confined to single-input, single-output layer stacks (as the name implies). In this model with the limited available dataset, the images are classified into three categories: normal, Pneumonia, and Covid. With the available data set, 251 images are taken as a training data set, and 66 are considered for the testing dataset. Table 1 has sample chest X-ray images of normal, covid, viral Pneumonia, respectively. We used 111 covid, 70 normal, and 70 viral pneumonia chest X-ray images from a total of 251training data sets. Similarly, we used 26 covid, 20 normal, and 20 viral pneumonia images in testing data set 66. Figure 3 depicts the outcome of the sequential model X-ray scans.

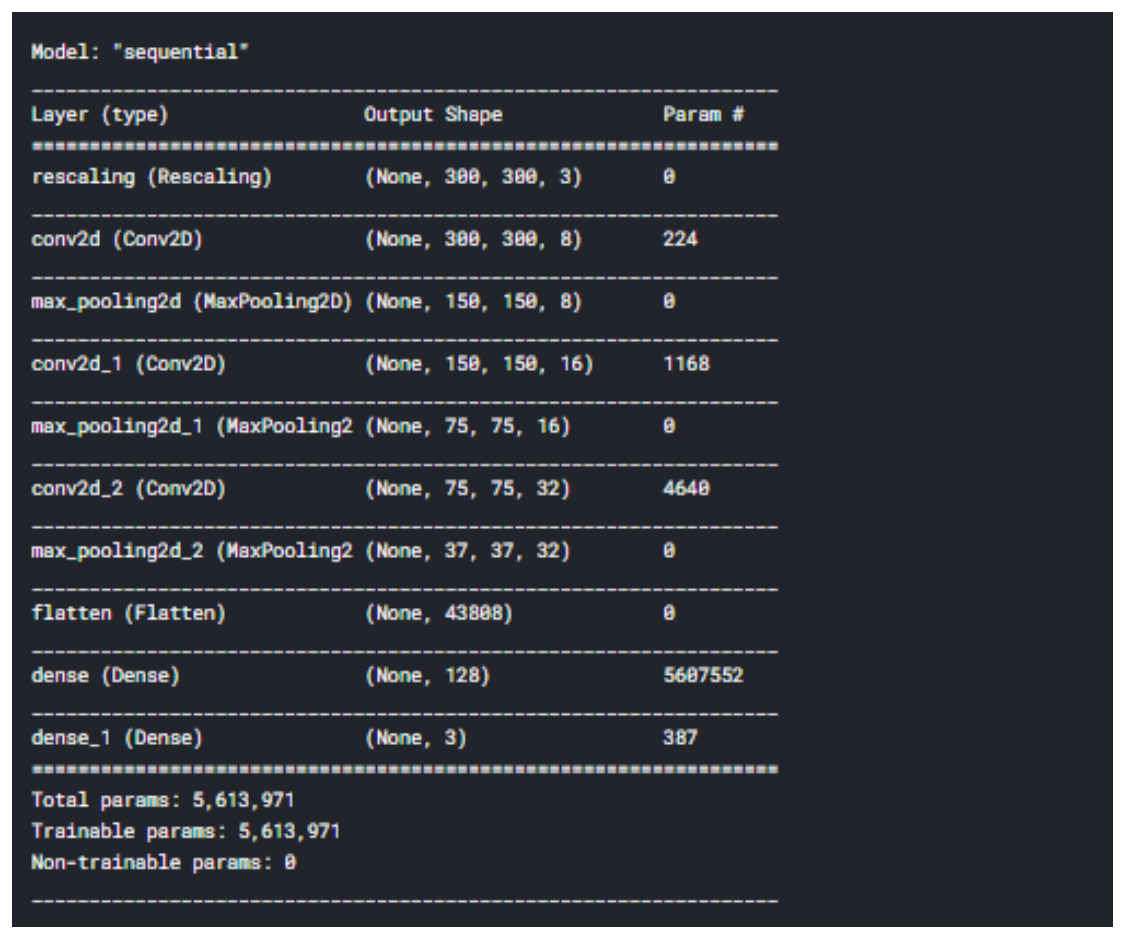

Figure 3. Model summary

\subsection{Performance analysis}

To evaluate the performance of the proposed CNN model we used the confusion matrix, which gives a holistic view of how well the model is performing as shown in Table 2. Using the confusion matrix, the precision (5), recall (6), F1-score (7), and accuracy (8) values were computed.

$$
\text { Precision }=\frac{\mathrm{TP}}{\mathrm{TP}+\mathrm{FP}}
$$




$$
\begin{aligned}
& \text { Recall }=\frac{\mathrm{TP}}{\mathrm{TP}+\mathrm{FN}} \\
& \mathrm{F} 1-\text { score }=\frac{2 \mathrm{XPXR}}{\mathrm{P}+\mathrm{R}} \\
& \text { Accuracy }=\frac{\mathrm{TP}+\mathrm{TN}}{\mathrm{TP}+\mathrm{TN}+\mathrm{FP}+\mathrm{FN}}
\end{aligned}
$$

Table 1. Sample chest X-ray image data set

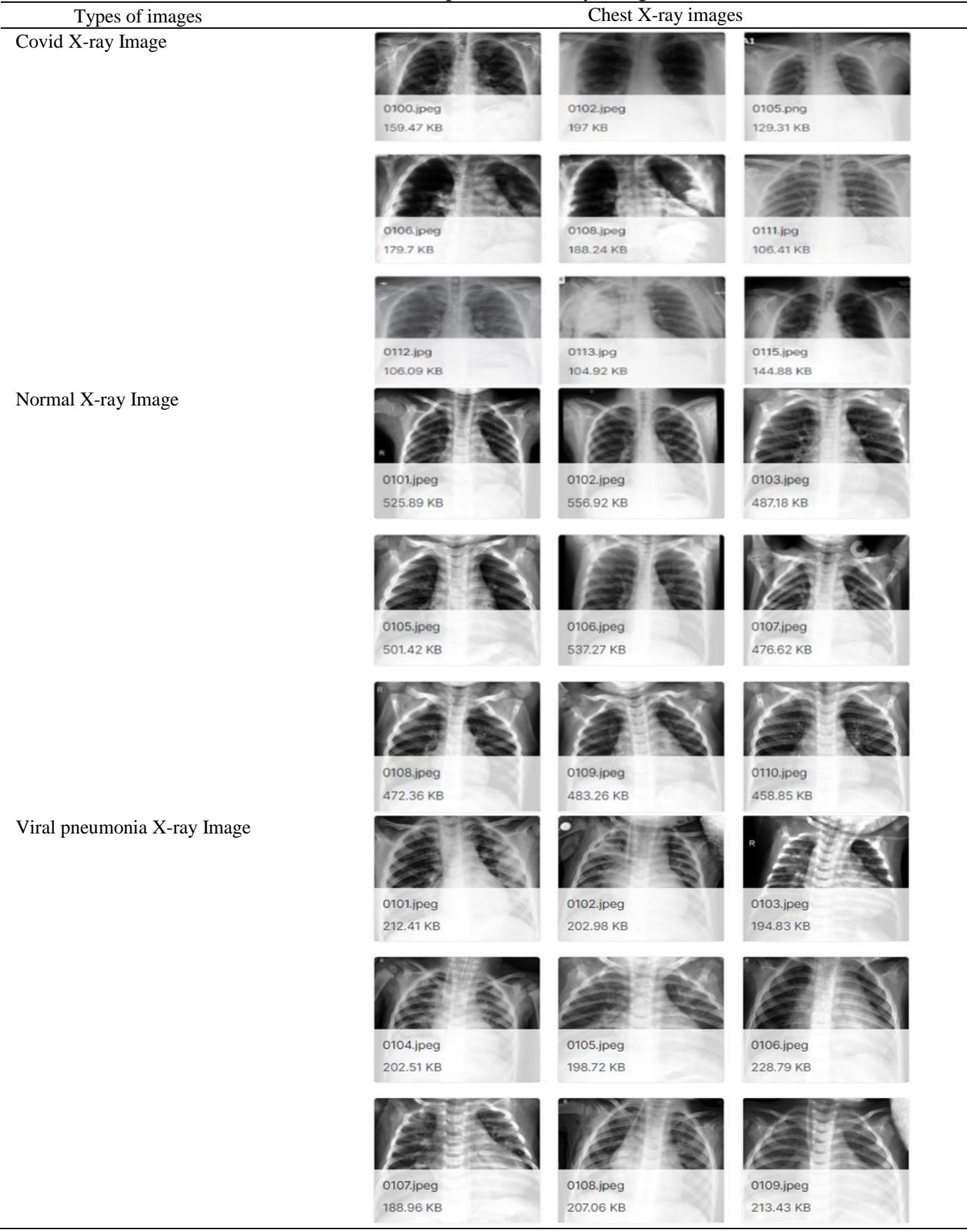

Table 2. Table confusion matrix

\begin{tabular}{ccc}
\hline & Predicted COVID-19 & Predicted non-COVID-19 \\
\hline Actual COVID-19 & True positive & False negative \\
Actual non-COVID-19 & False positive & True negative \\
\hline
\end{tabular}


The following graph shows the training and validation accuracy and loss as a function of epoch count. The variance in training and validation accuracy is extremely modest, about 15 epochs, and the loss disparity becomes more substantial after the 15th epoch, as seen in the graph. As a result, 15 epochs may be the optimum choice to avoid overfitting and an increase in loss. Figure 4 shows that the suggested model has a training accuracy of $91 \%$ and a training loss of 0.63 , as well as a validation accuracy of $81 \%$ and a validation loss of 0.7108 .
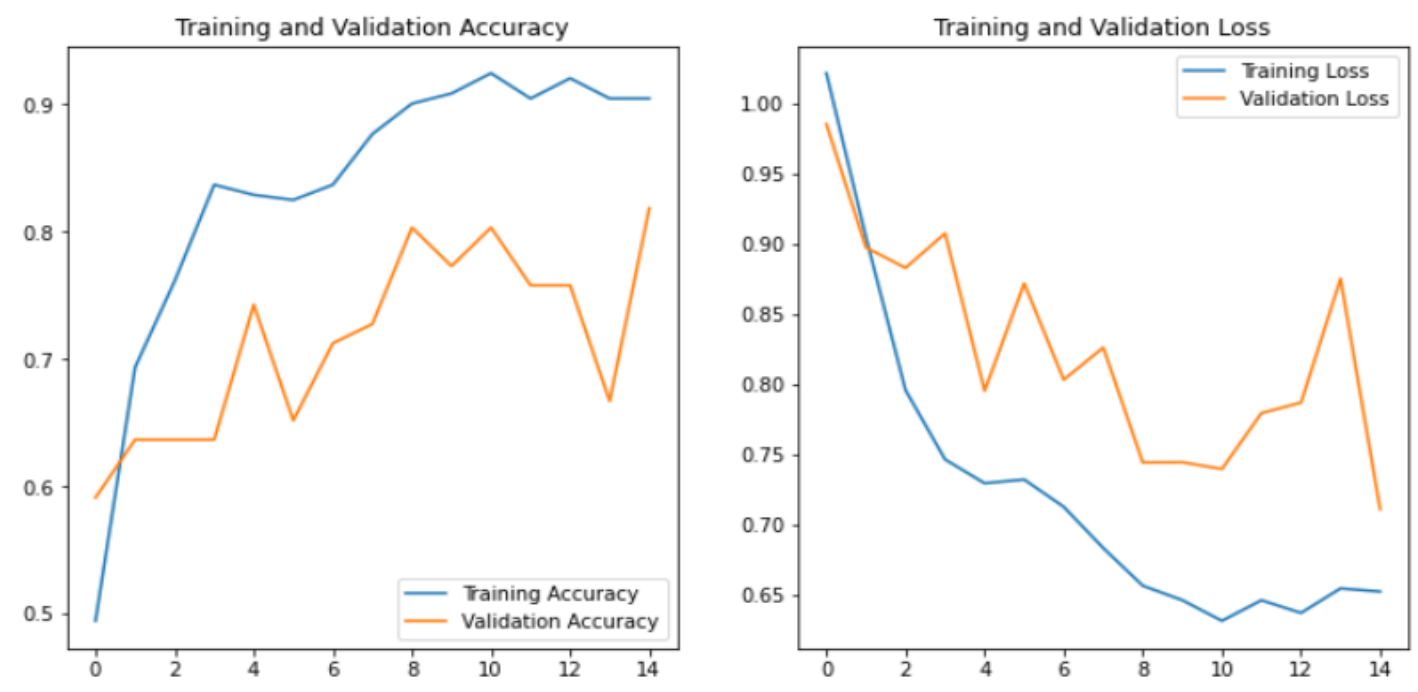

Figure 4. Training and validation (accuracy and loss)

\section{CONCLUSION}

This work sheds light on the early discovery of COVID-19 affected. The sparse categorical crossentropy method was used to categorize patients' chest X-ray images into three categories: covid, viral pneumonia, and normal. A method for integrating data preprocessing, rescaling, max pooling, and classification was described. We recognized in what way transfer knowledge might be useful for COVID-19 estimate. This archetypal may be used to diagnose COVID-19 affected from chest X-rays quickly and accurately. Our model has a $91 \%$ training accuracy and a training loss of 0.63 , as well as an $81 \%$ validation accuracy and a validation loss of 0.7108 . We want to add more CXR images from various hospitals to the collection in the future. We plan to look at using other clinical data and CXR images to improve prediction specificity and sensitivity even more. Because the IoT is quickly growing, the proposed model may be linked with it to give medical assistance to practitioners.

\section{ACKNOWLEDGEMENTS}

The author wishes to express her gratitude to anonymous reviewers for their insightful remarks and ideas, which significantly improved the quality of this paper.

\section{REFERENCES}

[1] A. S. Fauci, H. C. Lane, and R. R. Redfield, "COVID-19-navigating the uncharted," pp. 1268-1269, 2020, doi: 10.1056/NEJMe2002387.

[2] WHO, 2021. [Online]. Available: https://apps.who.int/iris/bitstream/handle/10665/331329/WHO-COVID-19laboratory-2020.4-eng.pdf.

[3] N. Chen et al., "Epidemiological and clinical characteristics of 99 cases of 2019 novel coronavirus pneumonia in Wuhan, China: a descriptive study," The Lancet, vol. 395, no. 10223, pp.507-513, Jan. 2020, doi: 10.1016/S01406736(20)30211-7.

[4] I. Walecka et al., "Psychological consequences of hospital isolation during the COVID-19 pandemic-research on the sample of polish firefighting academy students," Current Psychology, pp. 1-10, June. 2021, doi: 10.1007/s12144-021-01982-3.

[5] D. Wang et al., "Clinical characteristics of 138 hospitalized patients with 2019 novel coronavirus-infected pneumonia in Wuhan, China," JAMA, vol. 11, no. 323, pp.1061-1069, Feb. 2020, doi: 10.1001/jama.2020.1585. 
[6] F. Shi et al., "Review of artificial intelligence techniques in imaging data acquisition, segmentation, and diagnosis for COVID-19," IEEE Reviews in Biomedical Engineering, vol. 99, pp.1-1, 2020, doi: 10.1109/RBME.2020.2987975.

[7] H. Shi et al., "Radiological findings from 81 patients with COVID-19 pneumonia in Wuhan, China: a descriptive study," The Lancet Infectious Diseases, vol. 20 no. 4, pp. 425-434, April. 2020, doi: 10.1016/S14733099(20)30086-4.

[8] X. Xie, Z. Zhong, W. Zhao, C. Zheng, F. Wang, and J. Liu, "Chest CT for typical coronavirus disease 2019 (COVID-19) pneumonia: relationship to negative RT-PCR testing," Radiology, vol. 296, no. 2, pp. E41-E45, August 2020, doi: 10.1148/radiol.2020200343.

[9] X. Mei et al., "Artificial intelligence-enabled rapid diagnosis of patients with COVID-19," Nature medicine, vol. 26, no. 8, pp.1224-1228, May 2020, doi: 0.1038/s41591-020-0931-3.

[10] J. Lei, J. Li, X. Li, and X. Qi, "CT imaging of the 2019 novel coronavirus (2019-nCoV) pneumonia," Radiology, vol. 295, no. 1, pp.18-18, 2020, doi: 10.1148/radiol.2020200236.

[11] R. Ranjan, "Predictions for COVID-19 outbreak in India using epidemiological models," MedRxiv, April 2020, doi: 10.1101/2020.04.02.20051466.

[12] H. Y. F. Wong et al., "Frequency and distribution of chest radiographic findings in patients positive for COVID19," Radiology, vol. 296, no. 2, pp. E72-E78, May 2020, doi: 10.1148/radiol.2020201160.

[13] E. Prompetchara, C. Ketloy, and T. Palaga, "Immune responses in COVID-19 and potential vaccines: Lessons learned from SARS and MERS epidemic," Asian Pacific Journal of Allergy and Immunology, vol. 38, no. 1, pp. 19, 2020, doi: 10.12932/AP-200220-0772.

[14] M. E. Chowdhury et al., "Can AI help in screening viral and COVID-19 pneumonia?," IEEE Access, vol. 8, pp. 132665-132676, July 2020, doi: 10.1109/ACCESS.2020.3010287.

[15] L. J. Kroft, L. v. d. Velden, I. H. Girón, J. J. Roelofs, A. d. Roos, and J. Geleijns, "Added value of ultra-low-dose computed tomography, dose equivalent to chest x-ray radiography, for diagnosing chest pathology," Journal Of Thoracic Imaging, vol. 34, no. 3, pp.179, May 2019, doi: 10.1097/RTI.0000000000000404.

[16] M. E. Karar, D. R. Merk, V. Falk and O. Burgert, "A simple and accurate method for computer-aided transapical aortic valve replacement," Computerized Medical Imaging and Graphics, vol. 50, pp. 31-41, June 2016, doi: 10.1016/j.compmedimag.2014.09.005.

[17] A. Narin, C. Kaya, and Z. Pamuk, "Automatic detection of coronavirus disease (COVID-19) using X-ray images and deep convolutional neural networks," Pattern Analysis and Applications, vol. 24, pp. 1207-1220, May 2021, doi: 10.1007/s10044-021-00984-y.

[18] T. Ai et al., "Correlation of chest CT and RT-PCR testing for coronavirus disease 2019 (COVID-19) in China: a report of 1014 cases," Radiology, vol. 296, no. 2, pp. E32-E40, August 2020, doi: 10.1148/radiol.2020200642.

[19] S. Wang et al., "A deep learning algorithm using CT images to screen for Corona Virus Disease (COVID-19)," European Radiology, vol. 31, pp. 6096-6104, Feb. 2021, doi: 0.1007/s00330-021-07715-1.

[20] F. Sahar, R. Iqbal, H. Maha, and S. Salim, "Radiological Findings in Patients with COVID-19," Cureus, vol. 12, no. 4, Dec. 2020, doi: 10.7759/cureus.7651.

[21] S. Salehi, A. Abedi, S. Balakrishnan, and A. Gholamrezanezhad, "Coronavirus disease 2019 (COVID-19) imaging reporting and data system (COVID-RADS) and common lexicon: a proposal based on the imaging data of 37 studies," European Radiology, vol. 30, no. 9, pp. 4930-4942, April 2020, doi: 0.1007/s00330-020-06863-0.

[22] Wong et al., "Frequency and distribution of chest radiographic findings in patients positive for COVID-19," Radiology, vol. 296, no. 2, pp. E72-E78, May 2020, doi: 10.1148/radiol.2020201160.

[23] C. A. Raptis et al., "Chest CT and coronavirus disease (COVID-19): a critical review of the literature to date," American Journal of Roentgenology, vol. 215, no. 4, pp. 839-842, Oct. 2020, doi: 10.2214/AJR.20.23202.

[24] T. Ai et al., "Correlation of chest CT and RT-PCR testing for coronavirus disease 2019 (COVID-19) in China: a report of 1014 cases," Radiology, vol. 296, no. 2, pp. E32-E40, Feb. 2020, doi: 10.1148/radiol.2020200642.

[25] A. U. Ruby, P. Theerthagiri, I. J. Jacob, and Y. Vamsidhar, "Binary cross entropy with deep learning technique for image classification," International Journal of Advanced Trends in Computer Science and Engineering, vol. 9, no. 4, pp. 5393-5397, Aug. 2020, doi. 10.30534/ijatcse/2020/175942020.

[26] Z. Karhan and A. K. A. L. Fuat, "COVID-19 Classification Using Deep Learning in Chest X-Ray Images," In 2020 Medical Technologies Congress (TIPTEKNO), pp. 1-4, Nov. 2020, doi: 10.1109/TIPTEKNO50054.2020.9299315.

[27] I. Garafiev, G. Garafieva, A. Idiatullina, and E. Spirchagova, "Assessment of online education problems during the COVID-19 pandemic in Russia," Indonesian Journal of Electrical Engineering and Computer Science (IJEECS), vol. 23, no. 3, pp. 1689-1698, 2021, doi: covidwho-1404089.

[28] A. M. Saleh, H. Y. Abuaddous, O. Enaizan, and F. Ghabban, "User experience assessment of a COVID-19 tracking mobile application (AMAN) in Jordan," Indonesian Journal of Electrical Engineering and Computer Science (IJEECS), vol. 23, no. 2, pp. 1120-1127, August 2021, doi: 10.11591/ijeecs.v23.i2.pp1120-1127.

[29] F. M. J. M. Shamrat et al., "Sentiment analysis on twitter tweets about COVID-19 vaccines using NLP and supervised KNN classification algorithm," Indonesian Journal of Electrical Engineering and Computer Science (IJEECS), vol. 23, no. 1, pp. 463-470, July 2021, doi: 10.11591/ijeecs.v23.i1.

[30] M. A. Noori, S. A. S. Hussien, and T. A. Al-Janabi, "Blood donors appointment booking and managing system using PC and mobile web browsers in current pandemic (COVID-19)," Indonesian Journal of Electrical Engineering and Computer Science (IJEECS), vol. 23, no. 1, pp. 566-574, July 2021, doi: covidwho-1328280.

[31] H. M. Haglan, A. Sh. Mahmoud, M. H. AL-Jumaili, and A. J. Aljaaf, "New ideas and framework for combating COVID-19pandemic using IoT technologies," Indonesian Journal of Electrical Engineering and Computer Science (IJEECS), vol. 22, no. 3, pp. 1565-1572, June 2021, doi: 10.11591/ijeecs.v22.i3. 
[32] A. W. Reza, Md. M. Hasan, N. Nowrin, and M. M. A. Shibly, "Pre-trained deep learning models in automatic COVID-19 diagnosis," Indonesian Journal of Electrical Engineering and Computer Science (IJEECS), vol. 22, no. 3, pp. 1540-1547, June 2021, doi: 10.11591/ijeecs.v22.i3.pp1540-1547.

\section{BIOGRAPHIES OF AUTHORS}
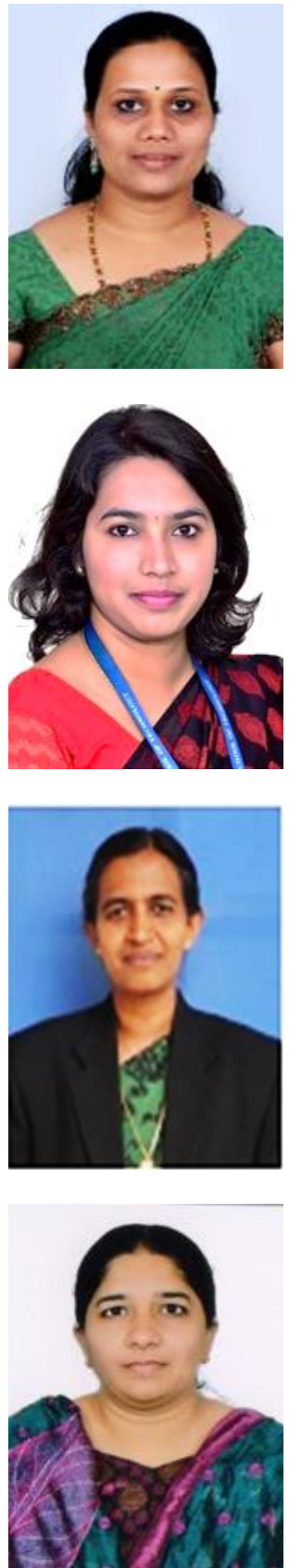

Chaithanya B. N., currently working as Assistant Professor in Department of Computer Science and Engineering at Gitam School of Technology, Bangalore. Areas of Interest are Network Security, Threat intelligence, Robotic process Automation and Cyber Security. Published papers in the those specified areas. Email Id: cnagaraj@ gitam.edu

Swasthika Jain T. J. currently working as Assistant Professor in Department of Computer Science and Engineering at GITAM School of Technology, Bangalore Campus. She is having 7.2 years of teaching experience. She is pursuing her PhD at GITAM School of Technology, Bangalore campus. She completed her Master degree (CSE) programme from Rajiv Gandhi Institute of Technology (VTU), Bangalore in 2013. She is Certified EMC Academic Associate from EMC Academic associate for data science and big data analytics and also Certified Associate in Python programming certification from the openEDG python institute. This is Cisco Networking academy course. Email: sjain@gitam.edu

A. Usha Ruby is an Associate Professor at Department of Computer Science and Engineering, GITAM (Deemed to be University), Bangalore, Karnataka, India. She received an M. Tech. degree from Anna University Chennai and Ph. D. from Bharath Institute of Higher Education and Research, Chennai in 2008 and 2017 respectively. Her research interests include Computer Networks, Digital Image Processing and Data Science. Email: uruby@ gitam.edu

Ayesha Parveen Currently working as Assistant Professor in the Department of Computer Science and Engineering at Gitam School of Technology, Bangalore. Areas of Interest are Data Mining, Machine Learning, Artificial Intelligence, and Cyber Security. Published papers in those specified areas. Email: aparveen@gitam.edu 